\title{
A HOLONIC CONTROL APPROACH FOR DISTRIBUTED MANUFACTURING
}

\author{
Paulo Leitão ${ }^{1}$, Francisco Restivo ${ }^{2}$

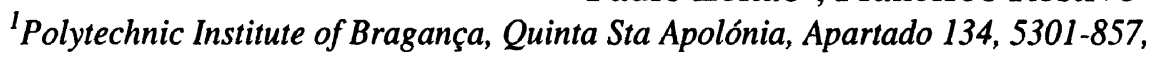 \\ pleitao@ipb.pt \\ ${ }^{2}$ University of Porto, Rua Dr. Roberto Frias, 4200-465 Porto, fjr@fe.up.pt
}

\begin{abstract}
Manufacturing systems are complex and often chaotic environments, being collated with customer satisfaction, products shorter life cycle and new ebusiness trends, becoming the design of manufacturing control applications a complex and hard task. As the traditional control approaches present serious problems to handle simultaneously the distribution of functions, global production optimisation, reaction to disturbances and reconfiguration, it is necessary to introduce new approaches that support the new intelligent manufacturing control systems. The paper introduces an innovative holonic manufacturing control architecture to face some of these unsolved problems by supporting an increase of flexibility and an agile reaction to disturbances without compromising the global production optimisation, being regulated by a coordination mechanism that allow fast reaction and adaptation to disturbances.
\end{abstract}

\section{INTRODUCTION}

Nowadays, manufacturing systems are complex, concurrent, asynchronous and often chaotic environments. Customer satisfaction, products shorter life cycle and new ebusiness trends lead to mass customisation and to the need of agile adaptation to manufacturing volatility. The manufacturing systems must support production of several types of products, being capable to change quickly from one product to another, demanding the execution of set-up configuration in the machines. These characteristics require efficient planning and control systems to optimise the production process through the elaboration of optimised production plans. However, the occurrence of disturbances, both machine failures and manufacturing modifications, implies deviations from the original plans. The manufacturing control system should react quickly to the disturbances elaborating alternative plans in order to minimize the effects of the disturbance in the system and the propagation of the disturbance outside the local system neighbourhood.

To support the complexity of manufacturing environments, there is an important research effort to develop innovative manufacturing control systems that increase the production process efficiency and optimisation, and react quickly to the occurrence of disturbances, such as described in (Van Brussel et al., 1998; Brennan et al., 1997; Parunak et al., 1998; Maturana and Norrie, 1996). 
This paper intends to introduce an innovative holonic manufacturing control architecture that intends to support an increase of flexibility and agile reaction to disturbances without compromising the global production optimisation. The proposed architecture defines new concepts in manufacturing control systems, using a hybrid control structure and distributing the control and scheduling among several decision levels, being regulated by a coordination mechanism that allow fast reaction and adaptation to disturbances.

\section{DISTURBANCE HANDLING PROBLEM}

The occurrence of a disturbance can lead to a deviation from the initial and optimised plan and to productivity decrease due to the machine/system inactivity. In this case the system should respond dynamically and quickly to the disturbance, using appropriate mechanisms according to the type of disturbance.

The main types of disturbance are the manufacturing or work order cancel, the machine failure, the introduction of a new manufacturing order, and the layout reconfiguration.

The manufacturing or work order cancellation comes from the need to abort the order due to the cancellation from the customer, a failure that provoked the destruction of the part, etc.. This disturbance causes a small impact in the system, because it is only necessary to release the operations already allocated and to reschedule the other operations in order to optimise the local schedule. The modification of the work order attributes, such as the change of temporal window to produce, may lead to the need to re-schedule all the operations.

A machine failure can occur due a tool collision, a tool broken, mistake in the machine programme, etc, causing a temporary or longer out of service of the machine, becoming incapable to accomplish the allocated work orders. The objective is to recover quickly the machine and in parallel to find out alternative solutions that minimize the deviation from the initial plan.

The introduction of a new manufacturing order, usually with high priority, requires the interaction between the entities in the system in order to schedule all operations of the task. This type of disturbance is only a problem when it leads to temporal conflicts with already allocated work orders.

The layout re-configuration implies the re-organization of the manufacturing resources, like the addition of the new resource or the remotion of a resource. The addition of a resource causes small impact in the system, because it increases the alternative solutions for the execution of manufacturing order, solving in some cases some conflicts problems. On the other hand, the remotion of a resource, leads to a more complex problem, that can introduce conflicts in the system.

\section{AGENT-BASED HOLONIC ARCHITECTURE FOR MANUFACTURING CONTROL}

The capability of current control architectures to adapt with agility and quickly to internal disturbances and external environment changes is poor. Due to the rigidity of the control architectures, the changes on the behaviour of the control system have 
impact in different levels of the control architecture. As a consequence, the time spent with programming and debugging, in case of re-configuration, is high.

The manufacturing research community is faced with the need to develop new and innovative manufacturing control systems, which present more agility and flexibility, and higher robustness against disturbances. In ADACOR architecture (Leitão and Restivo, 2001) the authors propose a new holonic approach to manufacturing control, implemented on a set of autonomous, intelligent and cooperative holons, each one representing a manufacturing entity (Leitão and Restivo, 2002).

\subsection{Internal Architecture of an ADACOR Holon}

The architecture is based on a set of holons grouped in operational, supervisor, product and task holon classes. The operational holon represents the physical manufacturing devices, such as tool machines, robots and automated guided vehicles. The supervisor holon coordinates several operational and supervisor holons, introducing coordination features and global optimisation in the system. The product holon represents the product data, such as the product model and the process model, and is responsible for the process planning. The task holon controls the execution of a manufacturing order and contains the associated dynamic information.

The generic ADACOR holon comprises the physical resource, capable to perform manufacturing tasks (if applicable), and the Logical Control Device (LCD) implemented using agent technology (Leitão and Restivo, 2002). This logical part of the holon comprises a local knowledge base, decision component (DeC), communication component (ComC) and physical interface component (PIC), as represented in Figure 1.

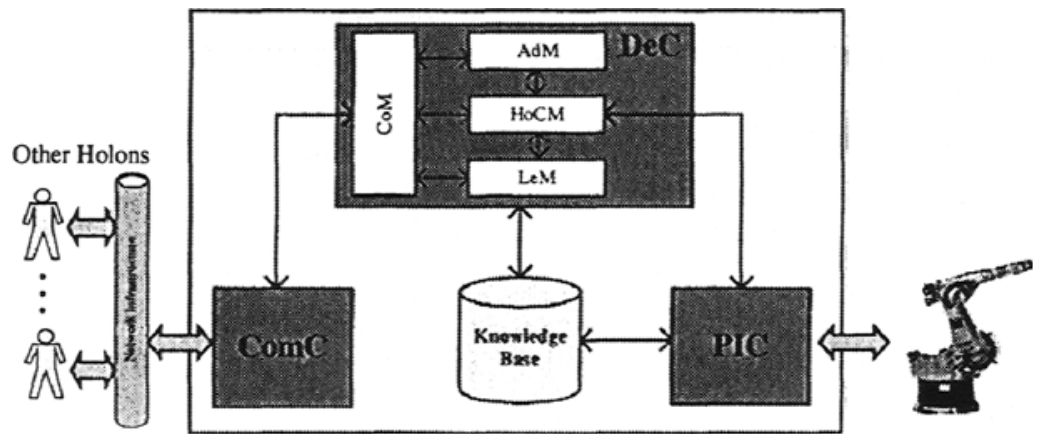

Figure 1 - Internal Architecture for an ADACOR Holon

The decision component, developed partially in a rule-based programming language, controls the activities of the holon and includes the holon control (HoCM), cooperation (CoM), adaptation (AdM) and learning (LeM) modules. The communication component deals with the interaction with other holons, making transparent the data exchange by using appropriate ontologies and the FIPA-ACL as communication language. The physical interface component comprises the mechanisms for the interaction with the physical devices that makes transparent the 
access to manufacturing resources. The local knowledge base stores all knowledge about the behaviour of the holon and the community where the holon belongs.

\subsection{Distributed Control Concept}

Aiming to achieve more flexible and agile features, the architecture proposes a distribution of the control by several decision-making levels, defining the local control, operational control and coordination control levels. The local controller level deals with the machine intrinsic control mechanisms and will not be described in this document.

\subsubsection{Operational Control Level}

Each operational holon in the architecture has abilities to perform local scheduling, dispatching, monitoring and reaction to disturbances functionalities.

The local scheduling module uses a scheduling engine and the local knowledge to implement the scheduling of the operations allocated to the holon. This module supports the optimised local schedule, the short term scheduling (when accepting the supervisor holon advices) and the agile reaction to disturbances. The local scheduling mechanism is a plug-in mechanism developed for the single machine scheduling problem, that uses a set of basic scheduling heuristics, such as EDD (Earliest Due Date) and SPT (Shortest Processing Time).

\subsubsection{Coordination Control Level}

The coordination control level introduces coordination in the control process, using the supervisor concept from bionic manufacturing systems. The coordinator entities are responsible for the global production optimisation, and are represented by supervisor holons. These supervisor holons, which can represent a manufacturing cell or a shop factory, have also a scheduling engine that deals with the multiple machines scheduling problem.

The optimised scheduling, elaborated at the coordination level, can be done in two different ways: task allocation process at supervision level or centralised scheduling. In the first approach, the supervisor holon launches several task allocation processes for the lower level, announcing the operations, and coordinates the negotiations in order to achieve the optimised schedule. In the second approach a centralised scheduling is executed by the supervisor holon using one algorithm for multiple machines problem.

The schedule elaborated by this holon eventually takes more time to achieve than the schedule obtained by each operational holon, but is optimised. The schedule elaborated is passed to the operational holons, in its coordination domain, as an advice.

\subsubsection{Distributed Scheduling}

There are scenarios where the control application can run without the presence of coordination levels, for example in heterarchical architecture or when there is a failure in the supervisor holon. The architecture defines a distributed and dynamic scheduling mechanism, Figure 2, which uses a multi-round Contract Net protocol 
(Smith, 1980) with some extensions, such as supporting multiple iterations and bid partial quantities.

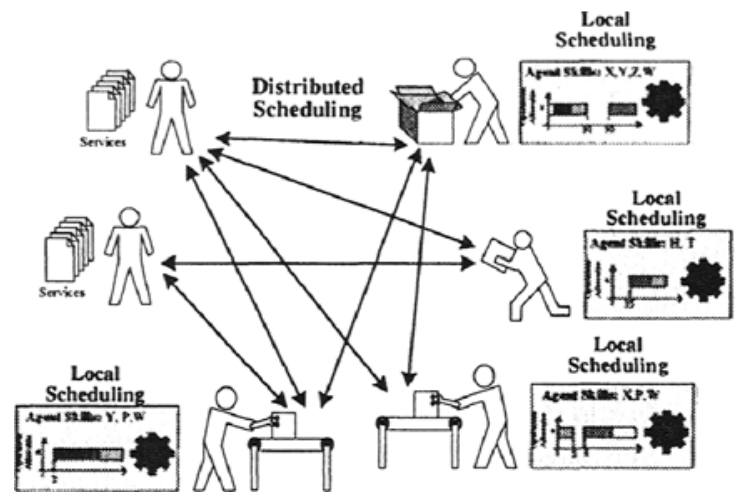

Figure 2 - Distributed and Local Scheduling

In this approach each operational holon has a local scheduling engine and is responsible for its own schedule, build from the local knowledge and goals. Operational holons verify the ability to execute the operation and find out their capacity to fulfil the operation due date before they submit their proposals. The global scheduling is achieved by the interaction between operational and task holons.

\section{THE ADAPTIVE CONTROL APPROACH CONCEPT}

The architecture allows different control approaches, such as hierarchical, federation and heterarchical, but in order to optimise the reaction to disturbances and the agility to unexpected events without compromising the global optimisation, it is necessary to evolve to a new control approach that combines those requirements.

The proposed control approach is based in holonic concepts, splitting the control into alternative states: steady state and transient state. In this holonic control approach the holons are organised in a federation structure and each holon has its own goals (the supervisor holons have the goal to achieve optimised schedule plans and operational holons the goal to optimise locally its behaviour) and evolves based in an autonomy degree, which is a dynamic factor that allows an operational holon to follow or not the advices sent by the supervisor holons.

\subsection{Steady State}

In the steady state, the supervisor holons that represent cells and/or the shop floor, have coordination functions through the elaboration of optimised schedule plans for the supervisor and/or operational holons in its coordination domain. The task holons interact with the supervisor holons, which will interact with other supervisor and operational holons, hierarchically in lower level, in order to allocate the manufacturing orders.

The supervisor holon elaborates optimised schedule for its coordinated resources, and dispatches it to the operational and supervisor holon that have enough 
autonomy to accept or reject the proposed schedule. If the operational holon rejects one or more proposed operations, the supervisor holon should re-schedule the production plan, trying to find alternatives, considering the previous rejection. If the operational holons accept the proposed operations, they are actualized in its local agenda.

\subsection{Transient State}

The need to react rapidly to a disturbance that requires the update to the original plans may not be satisfied by the steady state. In this case the system re-organise itself for a short period of time, with each operational holon adjusting its autonomy factor, applying a general mechanism that comprises three important steps.

Initially, the operational holon tries to recover locally the failure, increasing slightly the autonomy factor and re-scheduling its operations, in order to minimize the deviation to the original plan. In case of success, the operational holon reduces again the autonomy factor and the disturbance is recovered. If the operational holon cannot recover from the failure or it cannot fulfil the due date of an operation, the operational holon increases the autonomy factor, according with the type of disturbance, and disseminates the need for a re-organisation into a heterarchical organisational structure to other holons. In parallel the operational holon re-schedule the operations, interacting with the task holons if necessary to notify the disturbance.

After the recovery of disturbance the operational holon should synchronize its schedule with the optimised schedule, notifying the supervisor holon about the new schedule, and reducing again its autonomy factor. Since the autonomy factor is reduced the dissemination of disturbance is finished and the other holons doesn't sense anymore the occurrence of disturbance, reducing as well its autonomy factor, returning the system to the previous structure (hierarchical or federated form).

\subsection{Autonomy Degree Mechanism}

The autonomy degree of an operational agent evolves dynamically in order to adapt its behaviour to the external environment context. At moment, fuzzy logic and neural networks are being considered for the implementation of the autonomy factor mechanism.

In the stationary state, aiming the global production optimisation, the autonomy degree is low, allowing the operational agent to follow the schedule advices sent by the supervisor agents. The occurrence of a disturbance leads to an increase of the autonomy degree, decreasing the weight of the scheduling advices, in order to try to recover locally and quickly the disturbance.

The coordination between the holons, to perform the re-organisation to support disturbances, is performed using pheromone-like mechanisms. Thus, the need for reorganisation is disseminated to other supervisor holons, through the propagation and deposit of the pheromone to the neighbourhood supervisor holons, as represented in Figure 3. The holons associated to each supervisor holon perceive the need for reorganisation by sensing the pheromone, and increases its autonomy factor according with the type of disturbance, leading to an heterarchical organisation. 


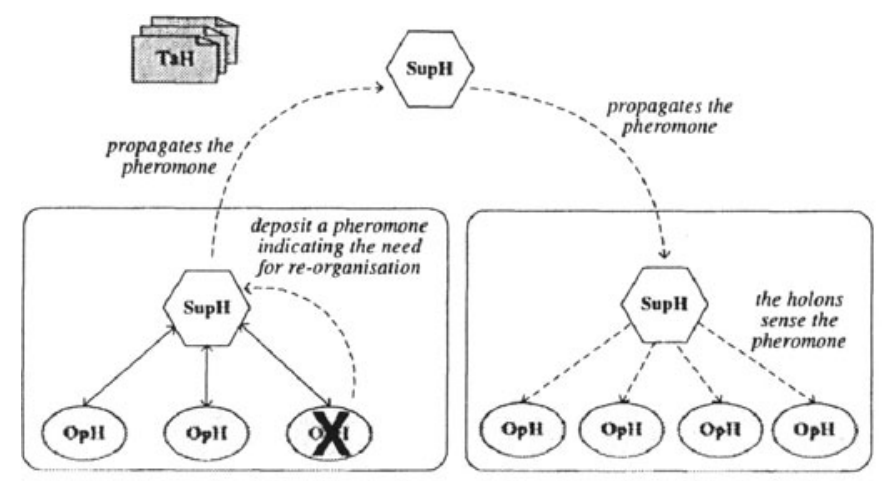

Figure 3 - Dissemination of a Disturbance

After the disturbance recovers, that should take short time, the autonomy degree decreases again in order to allow the operational holon to follow again the advices of the supervisor holons. Since the autonomy factor is reduced the dissemination of disturbance is finished and the other holons doesn't sense anymore the occurrence of disturbance, reducing as well its autonomy factor, returning the system to the previous structure.

The main advantage to use ant-based mechanisms to disseminate the need for reorganisation is to reduce the interaction level and communication overhead between the holons, improving the reaction to the re-organisation.

\section{COMPARISON WITH OTHER APPROACHES}

As referred before, other approaches for manufacturing control systems, such as PROSA architecture (Van Brussel, 1998), focus the agile reaction to disturbances. At this stage it is important to analyse some similarities and differences between the proposed architecture and the PROSA architecture.

PROSA is a reference architecture for manufacturing control and the ADACOR approach focus mainly in the distributed manufacturing shop floor control for job shop production type, with special attention to the agile reaction to disturbances, considering the set-up, maintenance and transportation operations.

In spite of both approaches have similar holon classes, the role of the holon responsible to introduce hierarchy is different in two approaches. The supervisor holon, used in ADACOR, coordinates several operational and/or supervisor holons, and implements the scheduling and control functions, representing manufacturing cell controllers or shop floor controllers. As the ADACOR approach supports federated control architectures, the supervisor holon coordinate the interactions between holons located in its coordination domain. In PROSA these functions are distributed by several staff holons, which doesn't have a specific role for cell controller. Additionally, in ADACOR approach the holons have learning capabilities in order to improve the performance of each holon during its life-cycle.

Both approaches use the holonic concepts to introduce more agility and flexibility in the reaction to disturbances maintaining the same levels of production optimisation. The solution adopted by both approaches is to introduce hierarchy in decentralised systems, and the dynamic re-configuration of the system. PROSA uses 
fixed rules and flexible strategies to change the control architecture supported by a meta-controller that define the basic re-configuration rules, while the ADACOR uses the autonomy degree mechanism associated to each operational holon to evolve between control architectures complemented by a pheromone-like mechanism to disseminate the need for the re-configuration.

\section{CONCLUSIONS}

The traditional manufacturing control systems have low capacity to adapt and react to the dynamic changes of its environment and to machine failures. The new generation of manufacturing control systems should comprise the high adaptation and reaction to the occurrence of disturbances and the optimisation of the global performance of the system, which require a global view of entire system.

This paper introduces an innovative holonic manufacturing control approach that intends to support an agile reaction to disturbances without compromising the production global optimisation and fulfilling the real time constraints.

The proposed approach to manufacturing control presents the following benefits: reconfiguration, flexibility, expansibility and fault tolerance. The reconfiguration capability is increased because the control approach allows a quick and agile reconfiguration to face the changes in production system strategy. The flexibility is increased due to the autonomy of the system entities and to the ability to support different organisational structures. In the ADACOR approach each component can be develop gradually and added to the system without stop or re-initialise the system, making easier the expansibility task. The reaction to disturbances is increased, because each holon is responsible for the execution of its tasks and for the recovery of the failures occurred in the resource that represents, minimizing the effects and propagation of the failure.

\section{REFERENCES}

1. Brennan R., Balasubramanian S. and Norrie D. A dynamic control architecture for metamorphic control of advanced manufacturing systems. In the Proceedings of the International Symposium on Intelligent Systems and Advanced Manufacturing, ed. by B. Gopaladrishnan, S. Murugesan, O. Struger, and G. Zeichen, 1997, pp. 213-223.

2. Leitão P. and Restivo F. Agent-based Holonic Production Control. To appear in Proceedings of $3^{\text {rd }}$ International Workshop on Industrial Applications of Holonic and Multi-Agent Systems (HoloMAS), 2-6 September, Aix en Provence, France.

3. Leitão P. and Restivo F. An Agile and Cooperative Architecture for Distributed Manufacturing Systems. In Proceedings of IASTED Robotics and Manufacturing International Conference. Cancun, Mexico, 21-24 May, 2001, Cancun, Mexico, pp 188-193.

4. Maturana, F. and Norrie D. Multi-Agent Mediator Architecture for Distributed Manufacturing, Journal of Intelligent Manufacturing, Vol. 7, 1996, pp. 257-270.

5. Parunak, H. Van Dyke, Baker A. and Clark S. The AARIA agent architecture: from manufacturing requirements to agent-based system design, Workshop on Agent-based Manufacturing, ICAA'98, 1998, Minneapolis.

6. Smith R.G. The Contract Net Protocol: High-Level Communication and Control in a Distributed Solver. In IEEE Transactions on Computers, Vol. C-29, No12, 1980, pp 1104-1113.

7. Van Brussel, H., Wyns J., Valckenaers P., Bongaerts L. and Peeters P. Reference Architecture for Holonic Manufacturing Systems: PROSA, Computers In Industry, vol. 37, 1998, pp. 255-274 